\title{
Jorge Luis Borges lector y prologuista de autores italianos
}

Palabras clave: Jorge Luis Borges, Biblioteca personal, prólogo, paratexto, letterato editore

El presente escrito procura analizar la actividad llevada a cabo por Jorge Luis Borges como prologuista y como colaborador de editoriales, en relación con tres obras de la literatura italiana incluidas en Biblioteca personal. En este contexto, ocupa un lugar central el concepto letterato editore, término elaborado por el italianista Alberto Cadioli (1995) para designar a los intelectuales que trabajan con las editoriales y dirigen colecciones editoriales ${ }^{1}$. Es un trabajo que tuvo gran importancia en Italia durante el siglo XX, especialmente desde la Segunda Guerra Mundial hasta los años ochenta, cuando según Cadioli tiene lugar el fin de la época del letterato editore ${ }^{2}$. Este puede actuar como un «filtro» y ser el «primer lector» de una obra, y también, como en el caso de las obras incluidas en Biblioteca personal, redescubrir algún libro olvidado ${ }^{3}$. En una declaración sobre Borges, Michel Lafon afirmó que una parte de la vasta y compleja actividad literaria de Borges se puede expresar con estos verbos: «Prologar, señalar, seleccionar, reunir, apadrinar, apoyar, reseñar, editar, dirigir, traducir» (Lafon, 1999). Según esto, la introdución de Borges en esta categoría está clara. Al igual

1 El letterato editore juega un papel determinante en la cultura, ya que elige los textos que se incluirán en el catálogo de la editorial. Las características más evidentes de esta figura son el vasto conocimiento de la literatura y el dominio de idiomas extranjeros. Un término que se puede añadir al letterato editore es el de iperlettore, también definido por Cadioli. En otras palabras: editores, escritores, críticos que actúan en nombre de una comunidad literaria (Cadioli, 2000: 31).

2 Véase el capítulo «La fine del letterato editore» (Cadioli, 1995: 197-205).

3 Como lo hiciera Leonardo Sciascia al asumir en el 1979 las tareas como director responsable de la colección editorial «La memoria» en la editorial Sellerio (Palermo). 
que autores italianos como Italo Calvino, Leonardo Sciascia, Giorgio Bassani, Elio Vittorini ${ }^{4}$, Borges vivió su vocación editorial y dedicó mucha atención a obras de otros autores5. Según Gian Carlo Ferretti, en el sector de los estudios del mundo editorial hay un vacío bibliográfico debido al prejuicio que nos lleva a separar la figura y la obra de un escritor con su contribución a las editoriales. Se tiende a subestimar el aporte de un intelectual a esta función por el hecho de oponer la creatividad como privilegio literario junto al trabajo editorial, entendido como actividad subordinada y dependiente de las editoriales (Ferretti, Guerriero, 2011:5). Este estudio quiere analizar un aspecto de la práctica editorial de Borges, el cual puede ser considerado como un letterato editore o un iperlettore, que colaboró no solo en el ámbito argentino, sino también en lo italiano, por su trabajo ocasional, en los años setenta, para el editor Franco Maria Ricci'. Entre Borges y los escritores italianos que trabajaron con las editoriales hay una ligera diferencia que merece la pena resaltar, que podría ser objeto de un estudio de mayor envergadura. Borges representa, de hecho, un caso aislado porque no se vincula a una editorial y principalmente porque no lo hace como trabajo continuado y estable. Mi hipótesis es que una relación simbiótica entre Borges y la cultura italiana está presente en la producción de elementos paratextuales específicos. Borges sería para los italianos un maestro en el arte de presentar un libro al lector; por otro lado, en sus prólogos se encuentran nuevas reflexiones sobre autores de la literatura italiana y sobre su poética. En un primer momento haré un breve resumen de la relación entre Borges y la cultura italiana; en segundo lugar, mencionaré libros que recopilan prólogos, y por último, intentaré aclarar algunas de las características del prólogo borgiano utilizando como ejemplo tres prólogos de Biblioteca personal que fueron escri-

4 A continuación, las referencias bibliográficas de mayor relieve sobre la actividad de estos escritores en relación al mundo editorial: Lombardo, G. (2008): Il critico collaterale. Leonardo Sciascia e i suoi editori. Milano: La Vita Felice; Ferretti, G. C., Guerriero, S. (2011): Giorgio Bassani editore letterato. San Cesario di Lecce: Manni; Clerici, L., Falcetto, B. (eds.) (1996): Calvino editore. Roma: Marcos y Marcos; Ferretti, G. C. (1992): L' editore Vittorini. Torino: Einaudi.

5 Esto se puede observar a través de las famosas palabras de Italo Calvino sobre el tiempo dedicado a los libros de los otros: «Il massimo del tempo della mia vita l'ho dedicato ai libri degli altri, non ai miei. Ne sono contento, perché l'editoria è una cosa importante nell'Italia in cui viviamo e l'aver lavorato in un ambiente editoriale che è stato di modello per il resto dell'editoria italiana, non è cosa da poco» (D'Eramo, Calvino: 1979).

6 A continuación se enumeran las obras de -o seleccionadas por-Börges publicadas por el editor Franco Maria Ricci (Parma) antes de comenzar a dirigir la colección «La biblioteca di Babele»: Borges, J. L., Bioy Casares, A. (1972): Cielo e inferno; Borges, J. L., Vázquez, M. E. (1973): Brume, dei, eroi; Borges, J. L., Bioy Casares, A. (1973): Racconti brevi e straordinari; Borges, J. L. (1974): Il congresso del mondo: illustrato con le miniature cosmologiche tantra. 
tos para obras italianas, siendo estas Il deserto dei Tartari de Dino Buzzati, los cuentos de Giovanni Papini (Il tragico quotidiano, Il pilota cieco y Parole e sangue) y Saggio su l'«Orlando Furioso» de Attilio Momigliano.

De acuerdo con la opinión del hispanista Roberto Paoli, entre Borges e Italia hubo un intercambio recíproco perfecto (Paoli, 1997: 1). Borges leyó literatura italiana y escribió sobre ella a lo largo de toda su vida. También ha sido el autor extranjero de mayor influencia en la literatura italiana del siglo XX (Paoli, 1997: 17)7. Según Italo Calvino, esto se debe a que los autores influenciados por Borges reconocieron en él una idea de literatura como un «mundo construido y gobernado por el intelecto» (Calvino, 1984). Una influencia de Borges en la cultura italiana se observa en la publicación de obras que reúnen formas literarias similares al prólogo (la solapa, la contraportada, el prefacio, la introducción) por los siguientes autores y críticos: Giorgio Colli, Elio Vittorini, Leonardo Sciascia, Italo Calvino, Roberto Calasso, Salvatore Silvano Nigro, Giacomo Debenedetti, Giorgio Manganelli ${ }^{8}$. Cuando se habla de formas paratextuales, hay más de un caso en el ámbito italiano en que se hace referencia de un modo más o menos explícito a Borges. La reciente recopilación de solapas escritas por Leonardo Sciascia durante su colaboración con la editorial Sellerio está introducida por un artículo escrito por el crítico siciliano Silvano Nigro

7 En relación con esto, léanse las palabras del crítico italiano: «Credo che l'influenza esercitata da Borges sulla narrativa italiana degli ultimi venti-venticinque anni sia la più estesa $\mathrm{e}$ feconda che sia venuta da uno scrittore straniero [...]. Il primo messaggio realmente influente sbarcato dall'America Latina [...] ha fatto presa in Italia e in Europa ribaltando il concetto erroneo che si aveva di quel subcontinente letterario, ritenuto a torto capace di esprimere soltanto scrittori politici o realisti più o meno magici». Esto coincide en parte con la opinión de Dario Puccini, por lo que Borges sería el único escritor, después de Kafka, en haber inaugurado un modo diferente de escribir e imaginar (Puccini, 1986). La bibligrafía italiana sobre Borges es extensa. Sobre la recepción de Borges en Italia véase el artículo de Elvira Dolores Maison (1992): «Algunos aspectos de la presencia de Borges en Italia» en Cuadernos Hispanoamericanos, 505/507, Homenaje a Jorge Luis Borges, 211-220. También hay que subrayar que Borges recibió en Italia muchos premios y homenajes: cabe recordar el «Premio Balzan» en 1981, la Laurea Honoris causa en la Universidad de Roma en 1984 y el «Premio Etruria» en 1985 . En los viajes que hizo en estas ocasiones conoció intelectuales como Sciascia, Montale, Calvino y Moravia.

8 A continuación se enumeran las primeras ediciones de las obras de los autores citados: Colli, G. (1983): Per una enciclopedia di autori classici. Milano: Adelphi; Vittorini, E. (1988): Risvolti dei Gettoni. Milano: Scheiwiller; Sciascia, L. (2003): Sciascia editore, ovvero la felicità di far libri. Palermo: Sellerio; Calasso, R. (2003): Cento lettere a uno sconosciuto. Milano: Adelphi; Calvino, I. (2003): Il libro dei risvolti. Torino: Einaudi; Nigro, S. S. (2007): L'arte del risvolto. Dieci note di Salvatore Silvano Nigro per dieci libri di Andrea Camilleri. Palermo: Sellerio; Debenedetti, G. (2012): Preludi. Palermo: Sellerio; Manganelli, G. (2016): Estrosità rigorose di un consulente editoriale. Milano: Adelphi. 
Salvatore bajo el título «Una specie collaterale della critica» (Sciascia, 2003: 7-27). La primera monografía dedicada al trabajo editorial de Sciascia lleva el título «Il critico collaterale» (nota n. 4). La introducción del editor y escritor Roberto Calasso en Cento lettere a uno sconosciuto, colección de solapas, empieza con una clara referencia a Borges: «Il risvolto [la solapa] è un'umile e ardua forma letteraria che non ha ancora trovato il suo teorico e il suo storico» (Calasso, 2003: 6)9. El prólogo introductorio escrito por Borges en 1975 en Prólogo con prólogo de prólogos dice: «Que yo sepa, nadie ha formulado hasta ahora una teoría del prólogo» (Borges, 1975: 8). Así como Borges asigna a la introducción de la recopilación el título Prólogo de prólogos, Calasso lo llama Risvolto dei risvolti.

Borges fue en períodos alternados poeta y narrador, pero siempre fue crítico y autor de ensayos, reseñas, notas y prólogos; corpus que consta de alrededor de mil textos (Ghezzani, 2008: 9). Aunque se trate de un autor bien conocido, vale la pena recorrer brevemente su producción como prologuista. El primer prólogo alógrafo es de 1925 para La calle de la tarde de Norah Lange (Buenos Aires: Ediciones J. Samet, 1925). En el mismo año en el prólogo de Inquisiciones -que en este caso es autorial (o autógrafo) - el argentino nos revela otra definición de este género: «la prefación es aquel rato en que el autor es menos autor. Es ya casi un leyente y goza de los derechos de tal: alejamiento, sorna y elogio. La prefación está en la entrada del libro, pero su tiempo es de posdata y es como un descartarse de los pliegos y un decirles adiós» (Borges, 1994: 7 ) $^{10}$. En los primeros cincuenta años, escribió alrededor de ochenta prólogos; en los

9 A continuación, el juicio completo de Calasso: «Per l'editore, spesso offre l'unica occasione per accennare esplicitamente ai motivi che lo hanno spinto a scegliere un certo libro. Per il lettore, è un testo che si legge con sospetto, temendo di incontrarvi un subdolo imbonitore. Eppure il risvolto appartiene al libro, alla sua fisionomia, come il colore e l'immagine della copertina, come i caratteri in cui è stampato. Eppure, una civiltà letteraria si riconosce anche dal modo in cui i libri si presentano. Lunga e tortuosa è stata la via percorsa dalla storia del libro prima di far nascere il risvolto» (Calasso, 2003: 6).

10 En 1927 y 1934 él redacta otras reflexiones sobre el género; véase el texto puesto al final del volumen Antología de la moderna poesía uruguaya 1900-1927: «¿Quién se anima a entrar en un libro? El hombre en predisposición de lector se anima a comprarlo -vale decir, compra el compromiso de leerlo- y entra por el lado del prólogo, que por ser el más conversado y el menos escrito es el lado fácil. El prólogo debe continuar las persuasiones de la vidriera, de la carátula, de la faja, y arrepentir cualquier deserción. Si el libro es ilegible y famoso, se le exige aún más. Se espera de él un resumen práctico de la obra y una lista de sus frases rumbosas para citar y una o dos opiniones autorizadas para opinar y la nómina de sus páginas más llevaderas, si es que las tiene» (Pereda Valdés, 1927: 219). En el prólogo escrito en 1934 para Reposo, de Elvira de Alvear, se lee: «Considero que la función del prólogo es establecer la discusión que debe suscitar todo el libro y evitar al lector las dificultades que una escritura nueva supone. En el libro común, el prefacio no tiene razón de ser, es un mero despacho de cortesías; en el excepcional, puede ser de alguna virtud» (De Alvear, 1934). 
últimos diez años de su vida alrededor de cien. En base a lo anterior, afirma Julio Chiappini: «No conocemos autor, al menos en lengua castellana, que sea tan obsequioso, tan prolífico como Borges en esto de decir prólogos» (Chiappini, 1991: 6) ${ }^{11}$. La primera colección, Prólogo con prólogo de prólogos (Borges, 1975), consta de treinta y ocho de los mismos, escritos entre 1925 y 1974. Posteriormente, en los setenta, Borges prologó cada uno de los veintinueve libros que eligió para su colección, «La biblioteca di Babele», dirigida por él a petición de Franco Maria Ricci; los prólogos fueron recogidos y publicados por Alianza en el volumen Prólogos de la Biblioteca de Babel, introducido por Antonio Fernández Ferrer (Borges, 2001). Por último, la colección «Biblioteca personal» por Hyspamérica Ediciones, ideada por Jorge Lebedev y dirigida en colaboración con María Kodama. En esta selección Borges se impuso la tarea de reducir a cien el número de sus preferencias literarias de las que, debido a su deceso, sólo consiguió sesenta y cuatro prólogos que fueron reunidos y publicados como obra autónoma por Alianza en 1988.

Más de una vez se ha señalado que Borges pertenece a la categoría de los escritores críticos pero alejados del mundo académico (Ghezzani, 2008: 9-12). A propósito de esto, según Dario Puccini: «Borges nunca ha sido ni ha pretendido ser crítico literario en el sentido «técnico» de la expresión y en el significado «funcional» de la misma» (Puccini, 1970: 145). Tomando las palabras del hispanista Tommaso Scarano, la de Borges es una crítica esencial, clara, muy agradable, educada, cortés, irónica, delicada, matizada (Scarano, 1998: 352); rasgos que aparecen quizás con más claridad aún leyendo los prólogos que, como sugiere Mariana Zinni, pueden ser leídos según la lógica del ensayo (Zinni, 2008). Alfonso Berardinelli define este tipo de escritura, la de los últimos prólogos, como un ejemplo de crítica lacónica, definición que también se presta para los italianos citados anteriormente. Que no se trata de elementos secundarios lo demuestra la publicación de estos textos en colecciones que tienen vida propia, que el lector puede leer con el mismo interés con el que se lee una colección de cuentos o ensayos ${ }^{12}$. Antes de ser escritor, Borges es un

11 El mismo concepto es retomado por el mismo Borges en sus diálogos con Osvaldo Ferrari donde se declara consciente de su vasta producción de prefaciones (Borges, Ferrari, 2003: 160).

12 Se ha hecho énfasis en repetidas ocasiones que la marginación de los paratextos borgeanos es solo aparente y que en efecto, no se trata de elementos marginales ni periféricos. Quisiera citar una opinión de Antonio Fernández Ferrer: «Para Borges no hay escrito secundario (pensemos en la importancia central que en sus textos tienen elementos tradicionalmente periféricos como epígrafes, posdatas, dedicatorias o notas e pie de página): su afán de escritura se entrega con parejo entusiasmo en cualquier texto. Podría hablarse, incluso, de una especie de irrenunciable «ética de la escritura» para la cual no existe «texto menor» [...]. Los 
atento lector y el prólogo es, en primera instancia, «un ejercicio de lectura», y también de memoria, con el objetivo de despertar el entusiasmo en quien se atreva a empezar un libro ${ }^{13}$. A Fernando Savater le impresionaron la «frescura antienfática del trazo» y en particular la «magia del enfoque» (Savater, 2005: 95) que el escritor, a pesar de sus ochenta y seis años, todavía logró expresar. Un factor evidente en los prólogos es la brevedad que, según Antonio Melis, se manifiesta como capacidad, a veces extraordinaria, de síntesis (Melis, 2005: 244 ${ }^{14}$. Según Ghezzani los escritos recogidos en Biblioteca personal serían menos representativos y tendrían una consistencia inferior a los de la primera fase (Ghezzani, 2008: 149). De hecho, la concisión que Borges se impuso no permite muchas posibilidades de argumentación. Según Chiappini, si hay «un denominador común en los prólogos éste es el encanto» (Chiappini, 1991: 7). Además, se encuentra una capacidad de seducir a través de frases sentenciadoras y de juicios de naturaleza estética. De la intertextualidad se hace un uso generalizado. Borges no puede evitar hablar de un texto o de un autor sin citar a otros: así que en los prólogos sobre las obras de Papini, Momigliano e Buzzati se citan Poe, Kafka, Bergson, Schopenhauer, Berkeley, William James, D’Annunzio, Dante y Croce. Un libro que recopila prólogos ofrece ante todo la perspectiva de un lector y se parece a una historia personal de la literatura, pero, sin confines geográficos, lingüísticos ni temporales. Borges casi siempre ha utilizado la palabra prólogo para distinguir esa tipología de texto de otros elementos peritextuales. Para intentar una teorización de este género hay que

prólogos de Borges [...] son cualquier cosa menos escritos meramente auxiliares o subsidiarios, y en ellos podemos encontrar tantas delicias como en cualquier otro texto de su autor» (Fernández Ferrer en Borges, 2001: 13-14).

13 En relación con esto, léase los siguientes pasos donde aparece claro cuánto había dado Borges a la actividad de leer: «Que otros se jacten de los libros que les ha sido dado escribir; yo me jacto de aquellos que me fue dado leer, dije alguna vez. No sé si soy un buen escritor; creo ser un excelente lector» (Borges, 1988: III). En los versos del poema Un lector: «Que otros se jacten de las páginas que han escrito; / a mí me enorgullecen las que he leído» (Borges, 1969: 151). La expresión está tomada del artículo de Mariana Zinni: «El prólogo es en primera instancia un ejercicio de lectura, un ensayo de lectura que funciona retroalimentando, que aparece antepuesto al texto y por lo tanto obliga a una lectura primera cuando en realidad es cronológicamente posterior al conocimiento del libro: es una manera de ejercer una política de lectura, ya que guía al lector por las páginas del libro obligándolo a veces a reconocer la propia lectura del prologuista» (Zinni, 2008: 181).

14 En esta escritura se consigue el «milagro estilístico» del que hablaba Italo Calvino en 1984 con respecto a Borges, maestro de la economía de la expresión y de la escritura breve, todo esto gracias a su capacidad de condensar, utilizando un fraseo cristalino y sobrio, una cantidad masiva de información en pocas páginas (Calvino, 1984). Los procedimientos literarios frecuentes son los mismos que se encuentran en otros textos del autor: enumeraciones, lítotes, antítesis, ironía, y citas exhibidas. 
concentrarse en los prólogos mismos, ya que, como afirma Lafon «el prólogo es el lugar de su propia teorización» (Lafon, 1999).

Así como Borges, por su clara voluntad de imprimir un marcado toque personal (Simson, 2008), está siempre presente en sus ensayos -que son el espacio para sus «obsesiones teóricas» (Ghezzani, 2008: 10)-, también está presente en sus prólogos. Leyendo Biblioteca Personal encontramos como protagonistas a Borges y a la literatura misma. Podemos identificar a los personajes de su biblioteca íntima y a la vez, reconocer algunos de los rasgos del retratista ${ }^{15}$. Esta subjetividad se observa en el hecho de que en cada prólogo Borges desplaza la atención a temas que le interesan abordar ${ }^{16}$. «En su vasta red de prólogos -como declara Echavarría (1983: 10)- se podría rastrear su intención literaria». Es el caso de la frase introductoria del ensayo de Momigliano, con la que justifica la idea de la lectura como placer hedonista y como forma de felicidad: «Juzgamos a los libros por la emoción que nos suscitan, por su belleza, no por razones de orden doctrinal o político», junto con la frase que se lee en el prólogo a Papini: «Fue un lector hedonista, siempre lo movió la dicha de leer, no un apremio de exámenes» (Borges, 1988: 43). Otro ejemplo es la siguiente frase referida a la Historia de la literatura italiana de Momigliano «En ella dijo que cada página de Gabriele D’Annunzio es una página de antología y lo dijo como un reproche». Borges en otros contextos había declarado su indiferencia ante D'Annunzio, culpable de querer impresionar al lector con cada palabra que a los ojos de Borges era un deseo infantil (Campra, 1982: 119). Además nos habla del descubrimiento de autores y libros, como en el siguiente caso: «Yo tendría diez años cuando leí, en una mala traducción española, Lo trágico cotidiano y El piloto ciego. Otras lecturas los borraron. Sin sospecharlo, obré del modo más sagaz. El olvido bien puede ser una forma profunda de la memo-

15 Queda bien explicado con las siguentes palabras de Goloboff: «El prologuista se explaya poco sobre los libros que presenta; prefiere dejar más lugar a los autores, a las ideas generales o especifícamente literarias que lo inquietaron. Pero no los presenta, y nos las presenta, a través de las ideas que él mismo tiene sobre las relaciones del escritor con el mundo físico, con el arte y, especialmente, con la literatura. De un modo mas tosco podría afirmarse que Borges no nos presenta obras, sino su modo de ver y leer esas obras, y que esa particularidad es la que mantiene la comunidad a que más arriba hice referencia Por esto, también más de una vez es difícil (si no inútil) distinguir cuándo Borges está afirmando algo de otro, y cuándo lo hace de sí mismo» (Goloboff, 1978: 255).

16 Enfoque confirmado por Borges en sus diálogos con Osvaldo Ferrari (Borges, Ferrari 2003: 160-161). En otra declaración se afirma que a veces los retratos de otros autores terminan siendo autorretratos de prologuista: «Ancora una volta è lecito chiedersi se il ritratto non sia piuttosto un autoritratto, cioè l'occasione per riaffermare alcuni motivi ricorrenti nella propria opera» (Melis, 2005: 250). 
ria» (Borges, 1988: 44) ${ }^{17}$. La biografía de los autores destaca por su concisión y se parece a las biografías sintéticas escritas por las reseñas de la revista «El Hogar», el la cual trabajó desde 1936 hasta $1939^{18}$ : «[Buzzati] fue periodista y se entregó después a la literatura fantástica» (Borges, 1988: 20); «Si alguien en este siglo es equiparable al egipcio Proteo, ese alguien es Giovanni Papini, que alguna vez firmó Gian Falco, historiador de la literatura y poeta, pragmatista y romántico, ateo y después teólogo. No sabemos cuál es su cara, porque fueron muchas sus máscaras» (Borges, 1988: 43); «Heredero de la áurea tradición de Croce y de De Sanctis, Attilio Momigliano se consagró al largo estudio y al grande amor de la literatura de Italia, que expuso en las universidades de Catania, de Florencia y de Pisa» (Borges, 1988: 124). La tendencia a enfatizar el elogio se debe a la idea de que el prólogo, género que «rebalsa de afecto» según palabras de Osvaldo Ferrari, (Borges, Ferrari, 2003: 160) debe ser tanto un estudio crítico como un homenaje (Borges, Ferrari 2003: 156). La parte inicial del texto que introduce Il deserto dei Tartari es halagadora y leída en un contexto italiano es aún más fuerte si se considera que en Italia -al contrario que en Francia o Alemania- Buzzati es un autor menor en las historias de la literatura ${ }^{19}$. Giovanni Papini en Italia ha sido también un autor de una alternada fortuna crítica y hay que destacar el hecho de que Borges mostró interés

17 Borges expresó la misma idea, esta vez relacionada con el conocimiento de la lengua latina en los versos del poema «Un lector»: «Mis noches están llenas de Virgilio; / haber sabido y haber olvidado el latín / es una posesión, porque el olvido es una de las formas de la memoria, su vago sótano, / la otra cara secreta de la moneda» (Borges, 1969: 151).

18 Según Gutierrez Girardot la biografía sintética no es un resumen cronológico-biográfico de la biografía del autor, «sino una selección de datos, cuya subjetividad se convierte en el apoyo de una interpretación, que a su vez da un sentido más preciso y profundo al concepto de biografía sintética» (Gutiérrez Girardot, 1992: 280). Como recalca Pérez, se trata de una manera no convencional lo que otros toman por típico: «Al reducir la vida a un solo momento que vale por fuerza simbólica, invierte el procedimiento habitual de la biografía que es contar toda la vida de una tal manera que el lector pueda entender al personaje y su obra en función de su pasado: su vida familiar, su medio ambiente, etc.» (Pérez, 1986: 226).

19 A continuación el incipit: «Podemos conocer a los antiguos, podemos conocer a los clásicos, podemos conocer a los escritores del siglo XIX y a los del principio del nuestro, que ya declina. Harto más arduo es conocer a los contemporáneos. Son demasiados y el tiempo no ha revelado aún su antología. Hay, sin embargo, nombres que las generaciones venideras no se resignarán a olvidar. Uno de ellos es, verosímilmente, el de Dino Buzzati (Borges 1988: 20)». En las historias de la literatura italiana del siglo XX su nombre normalmente aparece entre los autores menores; sin embargo Buzzati en otros paises es considerado un autor esencial: «Non può non apparire singolare che questo «minore» fuori d'Italia sia considerato, a torto o a ragione, un gigante della letteratura universale» (Giannetto 1996: 237). Sobre la fortuna de Buzzati en Francia y las razones de su poca consideración en Italia léase el siguiente artículo: Livi F., (1992), Traduire les classiques italiens, «Travaux du centre de traduction littéraire», n. 14, Université de Lausanne, pp. 31-42. 
por sus cuentos fantásticos ${ }^{20}$. De Momigliano, Borges aprecia la predisposición a redactar comentarios estéticos y la elección de este ensayo le permite incluir el Orlando Furioso («Laberinto de cristal» Borges, 1988: 125) en su biblioteca personal. Volviendo a Buzzati, Borges recuerda que los críticos italianos vieron en él la influencia de Poe y Kafka. Añade: «¿Por qué no aceptar sin desmedro alguno de Buzzati, ambos ilustres magisterios?» (Borges, 1988: 20) ${ }^{21}$. Es evidente que para Borges imitar a los autores del pasado sólo puede ser una fuente de orgullo y no una falta de originalidad. Asimismo, en estas páginas, Borges declara que su cuento El otro repite el argumento de un cuento de $\mathrm{Pa}-$ pini (Dos imágenes en un estanque).

Como se ha visto, el prólogo es tanto una excusa para hablar de una poética como un pretexto para hacer literatura; como afirma Lafon, «Llega un momento en que la novela no es más que el pretexto de su prólogo» (Lafon, 1999). Borges fue un lector y escritor que con un estilo identificable fue capaz de revitalizar un género literario, el prólogo. En el contexto cultural de Italia, este género, que se asume de forma diferente, nos dejó el legado de que la tarea de un escritor no consiste solo en escribir libros, sino que también consiste en promover y valorar la literatura en general.

\section{Bibliografía}

Berardinelli, A. (2012): «Debenedetti, ovvero la bella arte della critica laconica e senza fine». Il Foglio, 9 de junio.

Berardinelli, A. (2008): «La forma del saggio e le sue dimensioni». En: Giulia Cantarutti, Luisa Avellini, Silvia Albertazzi (eds.), Il saggio. Forme e funzioni di un genere letterario. Bologna: Il Mulino, 35-44.

Borges, J. L. (1969): Elogio de la sombra. Buenos Aires: Emecé.

Borges, J. L. (1975): Prólogo con prólogo de prólogos. Buenos Aires: Torres Agüero.

Borges, J. L. (1988): Biblioteca Personal. Madrid: Alianza.

Borges, J. L. (1994): Inquisiciones. Barcelona: Seix Barral.

Borges, J. L. (2001): Prólogos de la Biblioteca de Babel. Introducción de Antonio Fernández Ferrer. Madrid, Alianza.

20 Véanse también el prólogo que escribió para los cuentos publicados por Ricci: Papini, G. (1979), Lo specchio che fugge. Parma-Milano, F. M. Ricci.

21 Una comparación entre Borges y Buzzati propuesta por Olga Eggenschwiller Nagel: «La configuración del cosmos en la narrativa de Borges y Buzzati», en Quaderni iberoamericani, VII, ns, 55-56, Torino, 1982, pp. 339-363. 
Borges, J. L., Ferrari, O. (1986), Libro de Diálogos. Conversaciones de Jorge Luis Borges con Osvaldo Ferrari. Buenos Aires: Sudamericana. Ed. italiana (2003), Milano: Bompiani.

Cadioli, A. (1995): Letterati editori. Milano: Saggiatore.

Cadioli, A. (2000): L'editore e i suoi lettori. Bellinzona: Casagrande.

Calasso, R. (2003): Cento lettere a uno sconosciuto. Milano: Adelphi.

Calvino, I. (1979): «Intervista a Italo Calvino», por Marco D'Eramo, Mondoperaio, 6 de junio.

Calvino, I. (1984): «I gomitoli di Borges». La Repubblica, 16 de octubre.

Campra, R. (1982): L’America Latina, l'identità e la maschera. Roma: Editori Riuniti.

Chiappini, J. (1991): Los prólogos de Borges. Rosario: Zeus.

De Alvear, E. (1934): Reposo. Buenos Aires: Gleizer.

Echavarría, A. (1983): Lengua y literatura de Borges. Barcelona: Ariel.

Ferretti, G. C., Iannuzzi, G. (2014): Storie di uomini e libri. L'editoria letteraria italiana attraverso le sue collane. Roma: Minimum Fax.

Ferretti, G. C., Guerriero, S. (2011): Giorgio Bassani editore letterato. San Cesario di Lecce: Manni.

Genette, G. (1987): Seuils. Paris: Seuil.

Germán, Á., Rosato L. (2010): Borges, Libros y lecturas. Buenos Aires: Biblioteca Nacional.

Ghezzani, A. (2008): Borges critico letterario. Strutture e procedimenti discorsivi. Pisa: ETS.

Giannetto, N. (ed.) (1992): Il pianeta Buzzati. Atti del Convegno internazionale. Milano: Mondadori.

Goloboff, G. M. (1978): Leer Borges. Buenos Aires: Huemul.

Gutiérrez Girardot, R. (1992): «Crítica literaria y filosofía en Jorge Luis Borges». Cuadernos Hispanoamericanos, 505-507, «Homenaje a Jorge Luis Borges», 279-298.

Lafon, M. (1999): «Poética del prólogo». Boletín del Centro de Estudios de Teoría y Crítica Literaria, 7, 9-15.

Melis, A. (2005): «Il prologo come forma di appropriazione letteraria». En: Jorge Luis Borges (2005), Prologhi, con prologo ai prologbi. Milano: Adelphi, 242-252. 
Paoli, R. (1979): «La presenza della cultura Italiana nell'opera di Borges». L'Albero, 61-62, 70-90.

Paoli, R. (1997): Borges e gli scrittori italiani. Napoli: Liguori.

Pastormerlo, S. (1997): «Borges crítico». Variaciones Borges, 3, 6-15.

Pastormerlo, S. (2007): Borges crítico. Buenos Aires: Fondo de Cultura Económica.

Pereda Valdés, I. (1927): Antología de la moderna poesía uruguaya 1900-1927. Bueno Aires: El Ateneo.

Pérez, A. J. (1986): Poética de la prosa de Jorge Luis Borges. Hacia una crítica bakbtiana de la literatura. Madrid: Gredos.

Puccini, D. (1970): «Borges como crítico literario y el problema de la novela». En: El ensayo y la critica literaria en Iberoamérica. Toronto: Universidad de Toronto, $145^{-154}$.

Puccini, D. (1986): «Ma tutto è parola». Il Messaggero, 15. junio.

Savater, F. (2003): Borges. Bari: Laterza.

Scarano, T. (1998): «Ce vice impuni, la lecture». En: Jorge Luis Borges, Testi prigionieri. Milano: Adelphi, 341-354.

Simson, I. (2007): «Jorge Luis Borges e il rinnovamento della scrittura saggistica». En: Giulia Cantarutti, Luisa Avellini, Silvia Albertazzi (eds.), Il saggio. Forme e funzioni di un genere letterario. Bologna: Il Mulino, 315-332.

Todorov, T. (1984): Critique de la critique. Un roman d'apprentissage. Paris: Seuil.

Zinni, M. C. (2008): «Los prólogos de Borges: modos del devenir ensayo». Variaciones Borges, 25, 175-185. 
Nicola Tallarini

University of Graz

\section{Jorge Luis Borges reader and author of prefaces by Italian authors}

Keywords: Jorge Luis Borges, Biblioteca personal, preface, paratext, letterato editore

This essay focus on part of Borges' work as a prologue writer and as an editorcollaborator through the analysis of prologues he wrote to present these works to Argentinian readers. In the 1980s, Borges directed the editorial collection of the Biblioteca Hyspamérica in Argentina, where he included three works by Italian authors: Dino Buzzati's Il deserto dei Tartari, Giovanni Papini's short stories and Saggio su l'«Orlando Furioso» by Attilio Momigliano. The analysis centers on the work of an intellectual who enabled the modern reader to rediscover classic as well as forgotten works. The aim of the essay is to shed light on Borges' role as a letterato editore and as a prologuista. Furthermore, my analysis aims to confirm the hypothesis that there was a perfect symbiotic relationship between Borges and Italy. 
Nicola Tallarini

Univerza v Gradcu

\section{Jorge Luis Borges bralec in pisec predgovorov $\mathbf{k}$ delom italijanskih avtorjev}

Ključne besede: Jorge Luis Borges, Biblioteca personal, predgovor, paratekst, letterato editore

Pričujoči zapis se osredotoča na vidik Borgesovega delovanja kot pisca predgovorov in sodelavca založb, in sicer na podlagi analize predgovorov $\mathrm{k}$ trem delom italijanskih avtorjev, s katerimi jih je predstavil argentinskim bralcem. To so Il deserto dei Tartari Dina Buzzata, kratke zgodbe Giovannija Papinija in Saggio su l'»Orlando Furioso« Attilia Momiglianija, ki jih je Borges vključil v zbirko »Biblioteca personal«, katere urednik je bil v osemdesetih letih pri argentinski založbi Hyspamérica. Analiza se osredotoča na delo intelektualca, ki je modernemu bralcu omogočalo ponovno odkrivanje klasike in pozabljenih knjig. Članek prispeva k podobi Borgesa kot književno izobraženega urednika (letterato editore) in avtorja predgovorov ter potrditi hipotezo, da je med Borgesom in Italijo obstajala odlična simbiotična vez. 